PROCEEDINGS OF THE

AMERICAN MATHEMATICAL SOCIETY

Volume 139, Number 11, November 2011, Pages 3821-3829

S 0002-9939(2011)10804-9

Article electronically published on March 15, 2011

\title{
ESSENTIALLY FINITE VECTOR BUNDLES ON VARIETIES WITH TRIVIAL TANGENT BUNDLE
}

\author{
INDRANIL BISWAS, A. J. PARAMESWARAN, AND S. SUBRAMANIAN \\ (Communicated by Lev Borisov)
}

\begin{abstract}
Let $X$ be a smooth projective variety, defined over an algebraically closed field of positive characteristic, such that the tangent bundle $T X$ is trivial. Let $F_{X}: X \longrightarrow X$ be the absolute Frobenius morphism of $X$. We prove that for any $n \geq 1$, the $n$-fold composition $F_{X}^{n}$ is a torsor over $X$ for a finite group-scheme that depends on $n$. For any vector bundle $E \longrightarrow X$, we show that the direct image $\left(F_{X}^{n}\right)_{*} E$ is essentially finite (respectively, $F$-trivial) if and only if $E$ is essentially finite (respectively, $F$-trivial).
\end{abstract}

\section{INTRODUCTION}

For a smooth projective variety $X$ over a field of characteristic zero, the tangent bundle $T X$ is trivial if and only if $X$ is an abelian variety. This is not true for fields of characteristic $p>0$. Examples of varieties, different from abelian varieties, with trivial tangent bundle can be found in [4, [5] (see also 6]).

Let $X$ be a smooth projective variety, defined over an algebraically closed field of positive characteristic, with the property that $T X$ is trivial. Let $F_{X}: X \longrightarrow X$ be the absolute Frobenius morphism. For any $n \geq 1$, let $F_{X}^{n}$ be the $n$-fold composition of the self-map $F_{X}$.

Nori introduced the fundamental group-scheme [9, [10. We recall that after introducing the essentially finite vector bundles and then showing that they form a neutral Tannakian category, Nori defined the fundamental group-scheme to be the one given by this neutral Tannakian category. We also recall that a vector bundle $V$ on a smooth projective variety is essentially finite if and only if the pullback of $V$ by some projective surjective morphism is trivial. A special class of essentially finite vector bundles is the $F$-trivial vector bundle; a vector bundle is called $F$-trivial if its pullback by some power of the Frobenius morphism is trivial. The $F$-trivial vector bundles also define a neutral Tannakian category. The corresponding groupscheme is called the local fundamental group-scheme.

We prove the following proposition (see Proposition 2.5):

Received by the editors March 22, 2010 and, in revised form, September 15, 2010 and September $16,2010$.

2010 Mathematics Subject Classification. Primary 14L15, 14F05.

Key words and phrases. Essentially finite vector bundle, group-scheme, Frobenius morphism, tangent bundle. 
Proposition 1.1. Let $E$ be a vector bundle on a smooth projective variety $X$ with trivial tangent bundle. If $E$ is essentially finite, then the direct image $\left(F_{X}^{n}\right)_{*} E$ is essentially finite for every $n$.

If $\left(F_{X}^{n}\right)_{*} E$ is essentially finite for some $n$, then $E$ is essentially finite.

We also prove the following theorem (see Theorem 2.6):

Theorem 1.2. Let $X$ and $E$ be as in Proposition 1.1. If $E$ is $F$-trivial, then $\left(F_{X}^{n}\right)_{*} E$ is $F$-trivial for every $n$.

If $\left(F_{X}^{n}\right)_{*} E$ is $F$-trivial for some $n$, then $E$ is $F$-trivial.

The condition that $T X$ is trivial is used in the following three ways: All nonzero vector fields on $X$ are nowhere vanishing; the cotangent bundle $\Omega_{X}^{1}$ is a subbundle of a trivial vector bundle (equivalently, $T X$ is globally generated), and for a vector bundle $E$ on $X$, the Chern class $c_{1}(E)$ is numerically trivial if and only if $c_{1}\left(\left(F_{X}^{n}\right)_{*} E\right)$ is numerically trivial. More precisely, the crucial Lemma 2.1 is proved using the fact that all nonzero vector fields on $X$ are nowhere vanishing and that $T X$ is globally generated. In the proofs of Proposition 2.5 and Theorem 2.6 we use the fact that $c_{1}(E)$ is numerically trivial if and only if $c_{1}\left(\left(F_{X}^{n}\right)_{*} E\right)$ is numerically trivial.

\section{VARIETIES WITH TRIVIAL TANGENT BUNDLE}

Let $k$ be an algebraically closed field of characteristic $p$, with $p>0$. Let $X$ be an irreducible smooth projective variety defined over $k$ such that the tangent bundle $T X$ is trivial. Let $d$ be the dimension of $X$.

The $d$-dimensional vector space $H^{0}(X, T X)$ is equipped with the Lie bracket operation. Note that $H^{0}(X, T X)$ is a $p$-Lie algebra. There is a natural bijective correspondence between the $p$-Lie algebras over $k$ and the local group-schemes over $k$ of height one [8, p. 139]. Let

$$
G
$$

be the local group-scheme of height one corresponding to the $p$-Lie algebra $H^{0}(X, T X)$.

Let

$$
F_{X}: X \longrightarrow X
$$

be the absolute Frobenius morphism. For any integer $n \geq 1$, let

$$
F_{X}^{n}:=\overbrace{F_{X} \circ \cdots \circ F_{X}}^{n \text {-times }}: X \longrightarrow X
$$

be the $n$-fold iteration of $F_{X}$. By $F_{X}^{0}$ we will denote the identity morphism of $X$.

Lemma 2.1. The absolute Frobenius morphism $F_{X}$ defines a $G$-torsor over $X$, where $G$ is defined in (11).

Proof. Since $G$ corresponds to a Lie algebra of derivations on $X$, the group-scheme $G$ has a tautological action on $X$. Let

$$
q: X \longrightarrow Z:=X / G
$$

be the quotient morphism. We note that the action of $G$ on $X$ is free, because all the nonzero vector fields on $X$ are nowhere vanishing. Therefore, the above morphism $q$ defines a $G$-torsor on $Z$. 
It can be shown that the Frobenius morphism $F_{X}$ factors as

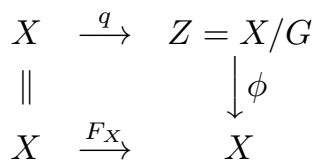

To prove this, let $\mathcal{E}$ denote the above principal $G$-bundle $q: X \longrightarrow Z$. Let $F_{Z}: Z \longrightarrow Z$ be the Frobenius morphism of $Z$. The pullback $F_{Z}^{*} \mathcal{E}$ is identified with the principal $G$-bundle obtained by extending the structure group of $\mathcal{E}$ using the Frobenius homomorphism $F_{G}: G \longrightarrow G$. We recall that $G$ is of height one, which means that the image of $F_{G}$ is the identity. Hence $F_{Z}^{*} \mathcal{E}$ is the trivial principal $G$-bundle $Z \times G \longrightarrow Z$. Therefore, we have a commutative diagram:

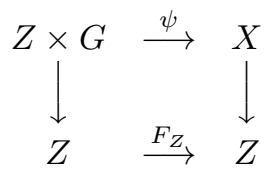

The morphism $\phi$ in (3) is the restriction of $\psi$ to the Cartesian product of $Z$ with the identity of $G$.

Now considering the scheme theoretic fibers of $q$ and $F_{X}$ we conclude that $\phi$ is an isomorphism.

Essentially finite vector bundles were defined in 9, 10. A vector bundle $E$ on a smooth projective variety $Y$ is essentially finite if and only if there is a projective variety $Z$ and a surjective morphism $\psi: Z \longrightarrow Y$ such that the pullback $\psi^{*} E$ is trivial [2, Theorem 1.1].

Corollary 2.2. The vector bundle $\left(F_{X}\right)^{*}\left(F_{X}\right)_{*} \mathcal{O}_{X} \longrightarrow X$ is trivial. In particular, the direct image $\left(F_{X}\right)_{*} \mathcal{O}_{X}$ is essentially finite.

Proof. From Lemma 2.1 it follows that $\left(F_{X}\right)^{*}\left(F_{X}\right)_{*} \mathcal{O}_{X}$ is the trivial vector bundle over $X$ with fiber $k[G]$ (see [8, p. 120 , Corollary 2]). Hence $\left(F_{X}\right)_{*} \mathcal{O}_{X}$ is essentially finite by the above criterion.

Lemma 2.3. Let $\gamma: Y \longrightarrow X$ be an étale cover. Then the tangent bundle of $Y$ is trivial.

Proof. Let $d \gamma: T Y \longrightarrow \gamma^{*} T X$ be the differential of the morphism $\gamma$. Since $\gamma$ is étale, we know that $d \gamma$ is an isomorphism. The vector bundle $\gamma^{*} T X$ is trivial because $T X$ is trivial. Hence the isomorphic vector bundle $T Y$ is trivial.

Lemma 2.4. Let $E$ be an essentially finite vector bundle over $X$. Then the direct image $\left(F_{X}\right)_{*} E$ is also essentially finite.

Proof. There is an étale Galois covering

$$
\gamma: Y \longrightarrow X
$$

and a positive integer $m$ such that $\left(F_{Y}^{m}\right)^{*} \gamma^{*} E$ is trivial, where

$$
F_{Y}: Y \longrightarrow Y
$$


is the absolute Frobenius morphism of $Y$ [10, Chapter II, Proposition 7] (see also [1, p. 557]). Since $\gamma$ is étale, the following diagram is Cartesian:

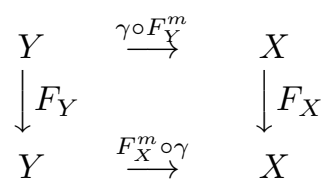

Hence

$$
F_{Y *}\left(F_{Y}^{m}\right)^{*} \gamma^{*} E=F_{Y *}\left(\gamma \circ F_{Y}^{m}\right)^{*} E=\left(F_{X}^{m} \circ \gamma\right)^{*}\left(\left(F_{X}\right)_{*} E\right)
$$

(see [3, p. 255, Proposition 9.3]).

Since $\left(F_{Y}^{m}\right)^{*} \gamma^{*} E$ is trivial, from Corollary 2.2 we know that $F_{Y *}\left(F_{Y}^{m}\right)^{*} \gamma^{*} E$ is essentially finite (note that Lemma 2.3 implies that Corollary 2.2 applies). Hence $\left(F_{X}^{m} \circ \gamma\right)^{*}\left(\left(F_{X}\right)_{*} E\right)$ is essentially finite by (5). This implies that the direct image $\left(F_{X}\right)_{*} E$ is essentially finite.

Proposition 2.5. For any essentially finite vector bundle $E \longrightarrow X$, and any $n \geq 1$, the direct image $\left(F_{X}^{n}\right)_{*} E$ is essentially finite. In particular, $\left(F_{X}^{n}\right)_{*} \mathcal{O}_{X}$ is essentially finite.

$A$ vector bundle $E$ on $X$ is essentially finite if $\left(F_{X}^{n}\right)_{*} E$ is essentially finite for some $n$.

Proof. Since $\left(F_{X}^{m+1}\right)_{*} E=\left(F_{X}^{1}\right)_{*}\left(F_{X}^{m}\right)_{*} E$, from Lemma 2.4 we conclude that $\left(F_{X}^{n}\right)_{*} E$ is essentially finite if $E$ is essentially finite.

To prove the second part, let $E \longrightarrow X$ be a vector bundle, and let $n$ be a positive integer such that the direct image $\left(F_{X}^{n}\right)_{*} E$ is essentially finite. Since $T X$ is trivial and $\left(F_{X}^{n}\right)_{*} E$ is essentially finite, it follows that $c_{1}(E)$ is numerically equivalent to zero.

The pullback $\left(F_{X}^{n}\right)^{*}\left(F_{X}^{n}\right)_{*} E$ is essentially finite because $\left(F_{X}^{n}\right)_{*} E$ is as well. Since $F_{X}^{n}$ is a finite morphism, the natural homomorphism

$$
\left(F_{X}^{n}\right)^{*}\left(F_{X}^{n}\right)_{*} E \longrightarrow E
$$

is surjective; hence $E$ is a quotient of the vector bundle $\left(F_{X}^{n}\right)^{*}\left(F_{X}^{n}\right)_{*} E$. As $E$ is a quotient of an essentially finite vector bundle and $c_{1}(E)$ is numerically equivalent to zero, we conclude that $E$ is essentially finite; see Remark 2.7 .

We recall that a vector bundle $E$ over $X$ is called $F$-trivial if the vector bundle $\left(F_{X}^{n_{0}}\right)^{*} E$ is trivial for some $n_{0}$.

Theorem 2.6. Let $E \longrightarrow X$ be an $F$-trivial vector bundle. Then for every $n$, the direct image $\left(F_{X}^{n}\right)_{*} E$ is $F$-trivial.

If $E$ is a vector bundle on $X$ such that $\left(F_{X}^{n}\right)_{*} E$ is $F$-trivial for some n, then $E$ is F-trivial.

Proof. To prove the first part, it suffices to show that $\left(F_{X}\right)_{*} E$ is $F$-trivial.

Let $n_{0}$ be an integer such that the vector bundle $\left(F_{X}^{n_{0}}\right)^{*} E$ is trivial. In (4), take $Y=X$ and set $\gamma$ to be the identity morphism of $X$. Now, from (5),

$$
F_{X *}\left(F_{X}^{n_{0}}\right)^{*} E=\left(F_{X}^{n_{0}}\right)^{*}\left(\left(F_{X}\right)_{*} E\right) .
$$

To prove that $\left(F_{X}\right)_{*} E$ is $F$-trivial, it is enough to show that $\left(F_{X}^{n_{0}}\right)^{*}\left(\left(F_{X}\right)_{*} E\right)$ is $F$-trivial. 
On the other hand, $F_{X *}\left(F_{X}^{n_{0}}\right)^{*} E$ is $F$-trivial if $F_{X *} \mathcal{O}_{X}$ is $F$-trivial, because $\left(F_{X}^{n_{0}}\right)^{*} E$ is a trivial vector bundle. Hence from (6) we conclude that $\left(F_{X}^{n_{0}}\right)^{*}\left(\left(F_{X}\right)_{*} E\right)$ is $F$-trivial if $F_{X *} \mathcal{O}_{X}$ is $F$-trivial. But $F_{X *} \mathcal{O}_{X}$ is $F$-trivial by Corollary 2.2, This completes the proof of the first part.

To prove the second part, let $E \longrightarrow X$ be a vector bundle of rank $r$, and let $n$ be a positive integer such that the direct image $\left(F_{X}^{n}\right)_{*} E$ is $F$-trivial. So there is a positive integer $m$ such that the pullback $\left(F_{X}^{m}\right)^{*}\left(F_{X}^{n}\right)_{*} E$ is a trivial vector bundle. Any pullback of a trivial vector bundle is trivial. Hence we may - and we will assume that $m \geq n$.

We saw in the proof of Proposition 2.5 that $E$ is a quotient of $\left(F_{X}^{n}\right)^{*}\left(F_{X}^{n}\right)_{*} E$. Hence the vector bundle $\left(F_{X}^{m-n}\right)^{*} E$ is a quotient of the trivial vector bundle

$$
\left(F_{X}^{m-n}\right)^{*}\left(F_{X}^{n}\right)^{*}\left(F_{X}^{n}\right)_{*} E=\left(F_{X}^{m}\right)^{*}\left(F_{X}^{n}\right)_{*} E .
$$

Define $V_{0}:=H^{0}\left(X,\left(F_{X}^{m}\right)^{*}\left(F_{X}^{n}\right)_{*} E\right)$, and let $\operatorname{Gr}\left(V_{0}, r\right)$ be the Grassmannian parametrizing quotients of $V_{0}$ of dimension $r$. Note that the evaluation of sections identifies $\left(F_{X}^{m}\right)^{*}\left(F_{X}^{n}\right)_{*} E$ with the trivial vector bundle $X \times V_{0}$. The quotient map

$$
\left(F_{X}^{m}\right)^{*}\left(F_{X}^{n}\right)_{*} E \rightarrow\left(F_{X}^{m-n}\right)^{*} E
$$

produces a quotient map $X \times V_{0} \rightarrow\left(F_{X}^{m-n}\right)^{*} E$. Therefore, there is a morphism

$$
f_{E}: X \longrightarrow \operatorname{Gr}\left(V_{0}, r\right)
$$

such that $\left(F_{X}^{m-n}\right)^{*} E$ is the pullback $f_{E}^{*} Q$, where $Q \longrightarrow \operatorname{Gr}\left(V_{0}, r\right)$ is the tautological quotient bundle.

Since $T X$ is trivial and $\left(F_{X}^{n}\right)_{*} E$ is $F$-trivial, it follows that $c_{1}(E)$ is numerically trivial. Hence $c_{1}\left(\left(F_{X}^{m-n}\right)^{*} E\right.$ ) is numerically trivial (this also uses the assumption that $T X$ is trivial). Since the line bundle $\operatorname{det} Q \longrightarrow \operatorname{Gr}\left(V_{0}, r\right)$ is ample and $c_{1}\left(\left(F_{X}^{m-n}\right)^{*} E\right)=c_{1}\left(f_{E}^{*} Q\right)$ is numerically trivial, it follows that $f_{E}$ is a constant morphism. Consequently, the vector bundle $\left(F_{X}^{m-n}\right)^{*} E=f_{E}^{*} Q$ is trivial. In particular, $E$ is $F$-trivial.

Remark 2.7. Let $E$ be an essentially finite vector bundle over a smooth projective variety $M$, and let $Q$ be a quotient bundle of $E$ such that $c_{1}(Q)$ is numerically trivial. Then $Q$ is also essentially finite. This can be derived from the definition of semistable bundles given in [10, p. 81] (note that this definition differs from the usual definition of semistability) and the definition of an essentially finite vector bundle given in [10, p. 82]. Here we have used the following characterization of an essentially finite vector bundle: There is an étale Galois covering

$$
\gamma: \widetilde{M} \longrightarrow M
$$

such that $\left(F_{\widetilde{M}}^{n}\right)^{*} \gamma^{*} E$ is trivial, where $n$ is some positive integer and $F_{\widetilde{M}}$ is the absolute Frobenius morphism of $\widetilde{M}$. The Chern class $c_{1}\left(\left(F_{\widetilde{M}}^{n}\right)^{*} \gamma^{*} Q\right)=\left(F_{\widetilde{M}}^{n}\right)^{*} \gamma^{*} c_{1}(Q)$ is numerically trivial because $c_{1}(Q)$ is as well. Since $\left(F_{\bar{M}}^{n}\right)^{*} \gamma^{*} Q$ is a quotient of the trivial vector bundle $\left(F_{\widetilde{M}}^{n}\right)^{*} \gamma^{*} E$ and $c_{1}\left(\left(F_{\widetilde{M}}^{n}\right)^{*} \gamma^{*} Q\right)$ is numerically trivial, we know that the vector bundle $\left(F_{\widetilde{M}}^{n}\right)^{*} \gamma^{*} Q$ is trivial; see the proof of the second part of Theorem 2.6. Consequently, $Q$ is essentially finite [10, 2, Theorem 1.1]. 


\section{The LOCAL FUndAMENTAL GROUP-SCHEME}

We continue with the set-up of the previous section. Let $X$ be ordinary.

Let $\left\{e_{1}, \cdots, e_{d}\right\}$ be a basis of the $k$-vector space $H^{0}(X, T X)$. Recall that $H^{0}(X, T X)$ is the Lie algebra of $G$.

Given a vector field $\theta \in H^{0}(X, T X)$, we have a vector field $\theta^{p}$ defined by

$$
\theta^{p}(f):=\overbrace{\theta \circ \cdots \circ \theta}^{p \text {-times }}(f)
$$

for all locally defined functions on $X$. Let

$$
\mu: H^{0}(X, T X) \longrightarrow H^{0}(X, T X)
$$

be the additive group homomorphism defined by $\theta \longmapsto \theta^{p}$. Note that

$$
\mu(c \theta)=c^{p} \mu(\theta)
$$

for all $c \in k$. Since $\mu$ is an additive group homomorphism and $\mu^{-1}(0)=0$, we conclude that $\mu$ is injective. Since

$$
\mu\left(\sum_{i=1}^{d} c_{i} \cdot e_{i}\right)=\sum_{i=1}^{d} c_{i}^{p} \cdot \mu\left(e_{i}\right)
$$

it follows that $\left\{\mu\left(e_{1}\right), \cdots, \mu\left(e_{d}\right)\right\}$ is a basis for the $k$-vector space $H^{0}(X, T X)$. Now from (9) it follows that $\mu$ is surjective. Therefore, $\mu$ is an isomorphism.

Recall the above basis $\left\{e_{1}, \cdots, e_{d}\right\}$ of $H^{0}(X, T X)$. Let $\left\{e_{1}^{*}, \cdots, e_{d}^{*}\right\}$ be the dual basis of $H^{0}(X, T X)^{*}$. For any $i \in[1, d]$, the element of the coordinate ring $k[G]$ of $G$ given by $e_{i}^{*}$ will be denoted by $x_{i}$. So

$$
k[G]=\frac{k\left[x_{1}, \cdots, x_{d}\right]}{\left(x_{1}^{p}, \cdots, x_{d}^{p}\right)} .
$$

Let

$$
x_{i} \longmapsto \sum_{j} f_{j}^{i} \otimes g_{j}^{i}
$$

be the co-multiplication structure of $k[G]$.

Define $G_{1}:=G$. Let $G_{2}$ be the group-scheme defined by the rule

$$
k\left[G_{2}\right]:=\frac{k\left[x_{1}^{1 / p}, \cdots, x_{d}^{1 / p}\right]}{\left(x_{1}^{p}, \cdots, x_{d}^{p}\right)}
$$

with the co-multiplication rule

$$
x_{i}^{1 / p} \longmapsto \sum_{j}\left(f_{j}^{i} \otimes g_{j}^{i}\right)^{1 / p},
$$

where $f_{j}^{i}$ and $g_{j}^{i}$ are defined in (10). We note that $G_{2}$ is a local group-scheme of height two.

Let $F_{G_{2}}: G_{2} \longrightarrow G_{2}$ be the absolute Frobenius morphism. Note that the image $F_{G_{2}}\left(G_{2}\right)$ coincides with the image of the natural homomorphism $G_{1}:=G \longrightarrow G_{2}$. More precisely, we have a short exact sequence of group-schemes:

$$
e \longrightarrow G_{1} \longrightarrow G_{2} \stackrel{F_{G_{2}}}{\longrightarrow} G_{1} \longrightarrow e .
$$


More generally, for any positive integer $n$, define the group-scheme $G_{n+1}$ as follows:

$$
k\left[G_{n+1}\right]:=\frac{k\left[x_{1}^{1 / p^{n}}, \cdots, x_{d}^{1 / p^{n}}\right]}{\left(x_{1}^{p}, \cdots, x_{d}^{p}\right)}
$$

with co-multiplication rule

$$
x_{i}^{1 / p^{n}} \longmapsto \sum_{j}\left(f_{j}^{i} \otimes g_{j}^{i}\right)^{1 / p^{n}} .
$$

Note that $G_{n}$ is a local group-scheme of height $n$. Let

$$
F_{G_{n}}: G_{n} \longrightarrow G_{n}
$$

be the absolute Frobenius morphism. The image $F_{G_{n}}\left(G_{n}\right)$ coincides with the image of the natural homomorphism $G_{n-1} \longrightarrow G_{n}$. As in (11), we have a short exact sequence of group-schemes:

$$
e \longrightarrow G_{1} \longrightarrow G_{n} \stackrel{F_{G_{n}}}{\longrightarrow} G_{n-1} \longrightarrow e .
$$

We will show that $G_{n+1}$ acts on $X$.

Given a vector field $D$ on $X$ and any positive integer $n$, let $D^{1 / p^{n}}$ be the vector field on $X$ defined by

$$
D^{1 / p^{n}}(f):=D\left((f)^{1 / p^{n}}\right)^{p^{n}}
$$

for all locally defined functions $f$ on $X$. So $D^{1 / p^{n}}=\left(\mu^{n}\right)^{-1}(D)$, where $\mu$ is the isomorphism in (7).

Let $S=\operatorname{Spec} k[t] / t^{2}$ be the Artin local $k$-algebra. Consider $G_{n+1}(S)$. We will construct an action of $G_{n+1}(S)$ on $X(S)$. A point of $G_{n+1}(S)$ is a $k$-algebra homomorphism:

$$
\frac{k\left[x_{1}^{1 / p^{n}}, \cdots, x_{d}^{1 / p^{n}}\right]}{\left(x_{1}^{p}, \cdots, x_{d}^{p}\right)} \longrightarrow k[t] / t^{2}
$$

Consider the homomorphism

$$
\frac{k\left[x_{1}^{1 / p^{n}}, \cdots, x_{d}^{1 / p^{n}}\right]}{\left(x_{1}^{p}, \cdots, x_{d}^{p}\right)} \longrightarrow k[t] / t^{2}
$$

defined by $x_{i}^{1 / p^{n}} \longmapsto t$. The action of this point of $G_{n+1}(S)$ on $X(S)$ is constructed as follows: The action of $\left(e_{i}\right)^{1 / p^{n}}$ sends a tangent vector $v$ to $v+\left(e_{i}\right)^{1 / p^{n}}$. (The tangent vector $\left(e_{i}\right)^{1 / p^{n}}$ is defined in (13); the tangent vectors $\left\{e_{i}\right\}$ are dual to $x_{i}$.)

The following diagram is commutative:

$$
\begin{array}{clr}
G_{n} \times X & \longrightarrow & X \\
F_{G_{n}} \times F_{X} \downarrow & & F_{X} \downarrow \\
G_{n-1} \times X & \longrightarrow & X
\end{array}
$$

where the morphisms $G_{n} \times X \longrightarrow X$ and $G_{n-1} \times X \longrightarrow X$ are the actions on $X$ of $G_{n}$ and $G_{n-1}$ respectively.

The quotient $X / G_{n}$ is identified with $X$, and the quotient morphism

$$
X \longrightarrow X / G_{n}
$$

coincides with the morphism

$$
F_{X}^{n}: X \longrightarrow X
$$


This is already proved for $n=1$ (see the proof of Lemma2.1). To prove the general case, use induction on $n$. The quotient by the subgroup-scheme $G=G_{1}$ in (12) is the Frobenius morphism $F_{X}$ (the case of $n=1$ ); hence from (12) it follows that the quotient by $G_{n}$ is the composition $F_{X}^{n}$.

Thus the morphism $F_{X}^{n}$ defines a principal $G_{n}$-bundle over $X$.

Fix a $k$-rational point $x_{0} \in X$. The local fundamental group-scheme $\varpi_{1}^{\text {loc }}\left(X, x_{0}\right)$ is defined to be the group-scheme associated to the neutral Tannakian category given by the $F$-trivial vector bundles on $X$ (see [7]); the definition of $F$-trivial vector bundles is recalled in Section 2. There is a tautological universal principal $\varpi_{1}^{\text {loc }}\left(X, x_{0}\right)$-bundle

$$
\widehat{X} \longrightarrow X
$$

Theorem 3.1. The local fundamental group-scheme $\varpi_{1}^{\text {loc }}\left(X, x_{0}\right)$ is the inverse limit of the group-schemes $\left\{G_{n}\right\}_{n \geq 1}$ constructed using the homomorphisms in (12). The tautological principal $\varpi_{1}^{\mathrm{loc}}\left(X, x_{0}\right)$-bundle $\widehat{X}$ in (15) is the inverse limit of the morphisms $F_{X}^{n}: X \longrightarrow X$.

Proof. Let $\mathcal{G}$ denote the inverse limit of the group-schemes $\left\{G_{n}\right\}_{n \geq 1}$ constructed using the homomorphisms in (12). Let

$$
E_{\mathcal{G}} \longrightarrow X
$$

be the principal $\mathcal{G}$-bundle defined by the inverse limit of the morphisms $F_{X}^{n}$ : $X \longrightarrow X$. From the commutativity of the diagram in (14) it follows that the inverse limit of the morphisms $F_{X}^{n}$ is a principal $\mathcal{G}$-bundle. Take any rational representation $V$ of $\mathcal{G}$. Therefore $V$ is a rational representation of $G_{n_{0}}$ for some $n_{0}$. Let

$$
E_{V}^{n_{0}} \longrightarrow X
$$

be the vector bundle associated to the principal $G_{n_{0}}$-bundle

$$
F_{X}^{n_{0}}: X \longrightarrow X
$$

for the $G_{n_{0}}$-module $V$. So the vector bundle $\left(F_{X}^{n_{0}}\right)^{*} E_{V}^{n_{0}}$ is trivializable. Thus $E_{V}^{n_{0}}$ is $F$-trivial. Consequently, we obtain a homomorphism,

$$
\rho: \varpi_{1}^{\text {loc }}\left(X, x_{0}\right) \longrightarrow \mathcal{G} .
$$

This also produces an isomorphism of the principal $\mathcal{G}$-bundle $E_{\mathcal{G}}$ with the principal $\mathcal{G}$-bundle $\widehat{X} \times^{\varpi_{1}^{\text {loc }}\left(X, x_{0}\right)} \mathcal{G}$ obtained by extending the structure group of the principal $\varpi_{1}^{\text {loc }}\left(X, x_{0}\right)$-bundle $\widehat{X}$ using the homomorphism $\rho$ in (16).

For the converse direction, let $E \longrightarrow X$ be a $F$-trivial vector bundle of rank $r$. Let $n_{0}$ be an integer such that the pullback $\left(F_{X}^{n_{0}}\right)^{*} E$ is trivializable. Fix an isomorphism of $\left(F_{X}^{n_{0}}\right)^{*} E$ with the trivial vector bundle $X \times k^{\oplus r}$. Using this trivialization, the natural action of $G_{n_{0}}$ on the fiber $\left.\left(\left(F_{X}^{n_{0}}\right)^{*} E\right)\right|_{\left(F_{X}^{n_{0}}\right)^{-1}\left(x_{0}\right)}$ defines a linear action of $G_{n_{0}}$ on $k^{\oplus r}$. Therefore, we obtain a homomorphism,

$$
\eta: \mathcal{G} \longrightarrow \varpi_{1}^{\text {loc }}\left(X, x_{0}\right)
$$

which is the inverse of $\rho$. 


\section{ACKNOWLEDGEMENTS}

We are very grateful to the referee for going through the manuscript carefully and providing detailed comments. The first- and third-named authors wish to thank the Kerala School of Mathematics, where a part of the work was carried out, for its hospitality.

\section{REFERENCES}

[1] I. Biswas and Y. I. Holla, Comparison of fundamental group schemes of a projective variety and an ample hypersurface, Jour. Alg. Geom. 16 (2007), 547-597. MR2306280 (2008g:14068)

[2] I. Biswas and J. P. dos Santos, Vector bundles trivialized by proper morphisms and the fundamental group scheme, Jour. Inst. Math. Jussieu (to appear), DOI:10.1017/S1474748010000071.

[3] R. Hartshorne, Algebraic Geometry, Springer Verlag, 1977. MR0463157 (57:3116)

[4] J.-i. Igusa, On some problems in abstract algebraic geometry, Proc. Nat. Acad. Sci. U.S.A. 41 (1955), 964-967. MR0074085 (17:534a)

[5] G. R. Kempf, Varieties with trivial tangent bundles, Topics in algebraic geometry (Guanajuato, 1989), 109-111, Aportaciones Mat. Notas Investigación, 5, Soc. Mat. Mexicana, México, 1992. MR1308335 (96a:14024)

[6] V. Mehta and V. Srinivas, Varieties in positive characteristic with trivial tangent bundle, Compos. Math. 64 (1987), 191-212. MR916481 (89e:14014)

[7] V. Mehta and S. Subramanian, Some remarks on the local fundamental group scheme, Proc. Ind. Acad. Sci. (Math. Sci.) 118 (2008), 207-211. MR2423233(2009c:14090)

[8] D. Mumford, Abelian Varieties, Oxford University Press, London, 1970. MR0282985 $(44: 219)$

[9] M. V. Nori, On the representations of the fundamental group scheme, Compos. Math. 33 (1976), 29-41. MR0417179(54:5237)

[10] M. V. Nori, The fundamental group scheme, Proc. Ind. Acad. Sci. (Math. Sci.) 91 (1982), 73-122. MR682517 (85g:14019)

School of Mathematics, Tata institute of Fundamental Research, Homi Bhabha ROAD, BOMBAy 400005, India

E-mail address: indranil@math.tifr.res.in

Kerala School of Mathematics, Kunnamangalam (PO), Kozhikode, Kerala 673571, INDIA

E-mail address: param_aj@yahoo.com

School of Mathematics, Tata Institute of Fundamental Research, Homi Bhabha ROAD, BOMBAy 400005, India

E-mail address: subramnn@math.tifr.res.in 